Article

\title{
Death, Entropy, Creativity and Perpetual Perishing: Some Thoughts from Whitehead and Stengers
}

\author{
Michael Halewood \\ Department of Sociology, University of Essex, Wivenhoe Park, Colchester CO4 3SQ, UK; \\ E-Mail: mhale@essex.ac.uk; Tel.:+44-1273-873-747
}

Academic Editors: Steve Fuller and Emilie Whitaker

Received: 28 June 2015 / Accepted: 18 August 2015/ Published: 28 August 2015

\begin{abstract}
In this paper, I argue that we need to rethink how we conceive of death as "inevitable". There are two main strands to my analysis. First, I use the work of Stengers to trace the complex and, occasionally, contradictory ways in which the concept of entropy was developed within physics in the 19th and 20th century. I argue that this has led to a general but ill-conceived notion of the universe as wasting away, as dying. This is a form of inevitability which has infected our understanding of what constitutes the death of individual humans. I then turn to the contrast that Whitehead draws between creativity and "perpetual perishing". I suggest that this contrast might help us to develop a wider, more coherent, approach to thinking about the status of death, and its supposed inevitability. In the final section, I reflect upon my father's death in 2013 in light of some of the concepts and problems raised throughout the paper.
\end{abstract}

Keywords: entropy; creativity; Whitehead; Stengers; inevitability; death

\section{Introduction}

Questions about extending or enhancing the lifecourse, through technoscience or any other means, would seem to be based on a tacit assumption that there is some kind of inevitability to death. This appearance of a necessity to death suggests that we are faced with the challenge of reconciling our lives to a specific kind of fate. In this article, I am not seeking to dismiss the idea that death is inevitable, but I do believe that it is important to consider how we think of this inevitability. Our concepts influence the responses that we are able to construct. Clearly, many cultures and societies have conceptualized death as being, in some way, "inevitable". However, this concept has taken on diverse forms, for example, in 
terms of a circle of life (and death), reincarnation, bodily resurrection and so on. One of my contentions is that, in the modern "West", we have tacitly accepted a concept of inevitability which is, in no small way, derived from an acceptance of ideas which were first formulated within science. I will therefore start by using the work of Stengers [1-3] to outline some of problems associated with the development of the concepts of entropy and irreversibility within modern physics. It is not a matter of evaluating whether such concepts are "right" or "wrong". Rather, it is a question of tracing how they have influenced the way in which we think about the inevitability of death in terms of an increase in disorder which characterizes the expansion of the universe. In the second part of this piece, I will use the work of Alfred North Whitehead to outline a different approach, one which insists that novelty and creativity require what Whitehead (borrowing from John Locke) terms "perpetual perishing". Overall, the aim of this article is to point to the need to tread carefully, and to realise how notions of life and death are intertwined both with each other and with wider concerns. To make this point, I will conclude with some reflections on my father's death in 2013.

\section{Is the Universe Dying?}

The question of whether the universe is dying might not be one that we ask every day. Yet, it is one which lurks in the background of our culture of thought insofar as we inhabit a world in which the pronouncements of science hold a certain sway. Most people today have some sense that the universe developed out of what is termed the "big bang". It is in this respect that the question of whether the universe is dying begins to make sense. If there were an original event in which energy was "created", and which leads to the expansion of the universe, ever outward, then it would seem reasonable to assume that this energy will at some point exhaust itself. The universe was, and is, cooling down, dissipating - it is dying. This is a vision of existence which peppers popular science books, newspapers and certain television programmes ${ }^{1}$.

In this article, I want to counter this ready acceptance of the idea that the universe is wasting away, is dying. I will use the work of Stengers [1-3] to trace the tortuous path of the development of the concept of entropy. The reason for this is to unsettle this version of inevitability which, I would suggest, has led us to envision real human mortality in a similar vein; as an inexorable wasting away of the physical energies of our bodies. This will involve a reappraisal of the second law of thermodynamics. The first law of thermodynamics states that in an event or reaction, energy is conserved. Energy is not created or destroyed. It moves from one form to another. This is linked to the second law which suggests that there is an inevitable increase in entropy which accompanies such events, reactions, and ultimately the universe. This is why the last of these can be thought of as "dying".

In the previous paragraph, I have used the word "entropy" without providing a definition of the term. This is because it is the very meaning and status of this concept which is at stake. Nevertheless, some initial orientation might be helpful. At a very general level, entropy appears to describe some physical

1 This question has become a little more complicated in recent years with the assertion, by physicists, that contrary to expectations, the expansion of the universe is not slowing down, indeed it is accelerating. This has led to claims that there is both "dark matter" and "dark energy" at work. These cannot be "seen", as yet, but these concepts help explain the increase in the rate in which galaxies are moving away from each other. Whether this is the case or not, is not important for the argument that I want to make here, as I hope will become clear. 
state. This is how physicists mainly use the term. In doing so, they claim to be measuring something which is represented by that physical state, namely the amount of disorder in a closed system. An increase in entropy is to do with an increase in disorder which also corresponds to a move toward equilibrium. Existence moves from the ordered to the disordered and an increase in entropy entails a dispersion or "flattening" of existence so that the complex bodies which make up the universe break down.

For example, if you strike a match in order to light a barbeque, the ordered energy contained in the head of the match is dissipated; it goes out into the world where it encounters a firelighter, perhaps. In turn, the ordered system of the firelighter then dissipates as it gives out the heat which cooks a burger. It might be going too far, at this stage, to state that the match or the firelighter die, but the wider conception of the transfer of energy as tied up with dissipation, with wasting away, is one which is intimately tied to the concept of entropy. The dispersed energy does not magically reform itself back into the match or the firelighter. Once it is gone, it is gone. As Stengers puts it: "entropy has continued to fascinate us by its message of fatality, by its association with loaded terms: degradation, heat-death, the arrow of time" ([2], p. 214). Furthermore, according to Stengers [2], this passive acceptance of the second law of thermodynamics is all too easy. This is not to say that the concept of entropy, and the second law of thermodynamics, are simply "wrong". Rather, we need to appreciate and understand the problems that led to the need for this law; what it presupposes, what it misses out. To provide these I will now summarize certain key points of Stengers' argument.

\section{The Question of the Second Law of Thermodynamics}

As stated previously, it is important to appreciate the force of the problem that the second law of thermodynamics was supposed to resolve. This does not mean re-opening the question in order to provide a more satisfactory answer, nor does it mean trying to denounce or deconstruct it. The term that that Stengers uses to describe her approach is "amanesis" ([2], pp. 182-88). This involves "dramatizing" the initial problem, immersing ourselves in its question, while bracketing the apparently simple solution which is nowadays taken for granted. A key element of this is the relation of thermodynamics to dynamics (or what we might call mechanics). These terms seem to be related as they both contain the word "dynamics". Classical mechanics (or dynamics) deals with questions of force, motion and velocity. Two of its major figures are Galileo and Newton. Taken in this light, it would then seem that the term "thermodynamics" is simply another branch, or an extension, of dynamics. The addition of the prefix "thermo-" appears to indicate that we are now in the realm of the mechanics (or dynamics) of heat. Stengers ([2], pp. 189-94) insists that we tread more carefully.

Within classical mechanics (dynamics), energy is not only conserved, it is technically reversible. This is the world of pendulums and clocks, for example. Early "physicists" such as Galileo were interested in explaining the ideal conditions and relations implicated in the swing of a pendulum. In order to accomplish this, the messiness of the actual world, with its friction and air resistance, needed to be overcome in order to develop equations which accounted for the movement of an ideal pendulum or falling body. Perhaps the most famous equation to come out of such endeavours is that of $\mathrm{F}=\mathrm{ma}$ (force equals mass times acceleration - this equation expresses Newton's second law of motion). The important point is that such equations are reversible. If you know the mass and acceleration, then you know the force. If you know the height that a pendulum reaches at the end its arc, then you know its starting point 
and the amount of force deployed. "If the instantaneous effect of a force is known, the force that determines it can be replaced by any force having an equal effect" ([2], p. 120). In this respect, the "ideal pendulum triumphs over the imperfect pendulum" ([2], p. 194) and, even more importantly, there is a "Galilean equality between cause and effect" ([2], p. 165). The past and the present are not so important in such considerations; the reversibility of the equations means that we can, in a sense, go backward and forwards in time, as what went before is conserved in what comes after.

In the early 19th century, thoughts turned form a world of ideal pendulums to understanding a world newly populated by strange beasts such as steam-engines. This introduced the problem of heat. In 1822 , Fourier developed his law of heat diffusion, stating that:

heat diffuses - there is no way of preventing or reversing it, in short of controlling it. On the contrary, in order to control heat, any conduction, any contact with bodies at different temperatures, must be avoided ([1], p. 37, Emphasis in original).

Unlike the reversible equations of classical mechanics, heat seems to introduce the irreversible and unavoidable problem of "heat loss". The diffusion of heat is, it seems, inevitable. As Stengers puts it:

This was the first intrinsically irreversible process to be given a mathematical expression, and it is this that caused a scandal: the unity of mathematical physics based on the laws of dynamics was shattered forever ([1], p. 37).

The scandal is that physics seems to have to give up its claim to provide pure equations which account for the motion of all the bodies in the universe. The inevitable heat loss which accompanies such events seems to imply that the reversibility implied by the equations of classical mechanics is impossible when it comes to questions of heat. Once a match is struck or a firelighter lit, it is impossible to collect all the "pieces" and put them back together into their original form. It is within this problematic realm that the laws of thermodynamics were formulated.

One of the original, and still influential, theorists of thermodynamics was Sadi Carnot (1796-1832). Carnot aimed to describe how heat "flowed" (and was related to work) by outlining the ideal operation of what he called a "heat-engine". This model (which is impossible in reality) is still used in teaching undergraduates today, even though Carnot maintained that the flow of heat was due to the movement of a "calorific fluid"-something we do not nowadays believe. Nevertheless, Carnot provides a model of the relation between the "flow" of heat and the volume and pressure of a liquid or gas. He describes the optimal conditions for this flow and for its transformation into work without any loss of energy. His heat-engine describes the ideal situation. Crucially, this engine is reversible, in the same sense that the equations of classical mechanics are. There is nothing which insists or forces the process to go in one direction only. Carnot, therefore, provided a "pure" description of the transmission of heat. It also led to the situation where "physics will transform itself into the prophet of the irreversible evolution toward heat-death, the state where no work is any longer possible" ([1], p. 197). This mention of "heat-death" indicates one of the main concerns of this article. The process by which "physics will transform itself" signals a main aspect of Stengers' interest throughout the two volumes of Cosmopolitics [2,3], namely, the changing status of physics in the 19th and 20th century. Although this is not the main focus of the argument being set out here, it is still necessary to follow Stengers' argument with regard to this a little further. 
The reason that the status of physics is important is that it involves the question not only of what physics studies but how it studies it. The questions of classical mechanics, of falling apples, rolling balls, pendulums, seem to be all around us. We can provide satisfactory, pragmatic, accounts of these by paying due attention to the forces and motions involved. There is a subtle difference with regard to heat. When things such as apples, balls, pendulums are put to work (to make cider or a clock), we know that, no matter how carefully we construct our devices, there will be a loss which seems to get in the way of us understanding "heat" in itself, without it being tied down by these "inevitable" losses. Carnot tries to avoid this difficulty by outlining an ideal heat-engine. But when he does so, he tries to exclude all elements of waste. In doing so, he develops "a set of ruses, that seek to minimize irreversible conduction" ([1], p. 37). To avoid the irreversibility associated with heat loss, Carnot outlines an ideal heat-engine which makes thermodynamics "reversible". This has important consequences for our current understanding of entropy. Our predominant and enduring concept of entropy, of heat-death, of the universe dying, of the inevitability of death as energy loss, is predicated upon an ideal, impossible, account ${ }^{2}$. We have not fully got beyond a definition which relies upon Carnot's ideal, one which is, by definition, reversible, not irreversible. This means that "the fact that irreversible energy transformations always result in an increase in entropy is simply another way of saying that they are always defined as a loss with respect to the reversible ideal" ([2], p. 214).

This is not the endpoint of Stengers' argument. Throughout the following sections of Cosmopolitics (Volumes I and II), she traces the complex and peculiar ways in which physics developed and transformed itself into 20th century theoretical physics. This involves the rise of atoms, quantum mechanics, as well as the work of Prigogine on "the arrow of time", irreversibility, equilibrium and "dissipative structures" ([3], pp. 105-204). Many of these arguments refer to the problem of entropy and equilibrium, although there is not space to deal with all these here. My interest is limited to the development of a notion of entropy as an irresistible path toward heat-death, and as something that supposedly suffuses the universe. To clarify this point, it is necessary to consider how we came to think of the universe as a singular entity, one which could undergo such an experience of inexorably wasting away.

Although Stengers does not put it exactly in these terms, it might be possible to argue that the modern concept of the universe only came into being with Einstein; more specifically with his equation regarding energy, mass and the speed of light $\left(E=\mathrm{mc}^{2}\right)$. As opposed to Newton's equation ( $\mathrm{F}=\mathrm{ma}$ ) which attempts to describe the interrelations of all the bodies within the universe. Einstein's equation subjects the universe as a singular entity to its scope. Or, as Stengers puts it: "a homogenous and isotropic universe is one of the rare objects simple enough to be explicitly treated using Einstein's equations" ([2], p. 206). This is an important point, as it apparently enables us to treat of the universe as one thing which inexorably and inevitably is heading towards heat-death, as a result of entropy. Treating the universe as singular, as almost observable, without accepting the particular strictures of a certain perspective, such as that of a physicist engaged in a specific problem, runs the risk of falling foul of what Haraway has called the "god-trick" ([4], pp. 188-96). This involves developing an abstraction without perspective,

2 In Volume II of Cosmopolitics, Stengers [3] outlines the robust attempts of her some-time collaborator, Ilya Prigogine, to develop a "modern" concept of entropy which avoids Carnot's starting point and problem. However, as Stengers makes clear, not only have Prigogine's concepts not succeeded in displacing or disrupting the traditional concept of entropy, as envisaged by most current physicists, it has not affected the way this concept operates in the background of our general thought. 
thereby making it apparently possible to make general claims, such as one regarding the universe as wasting away, as inexorably dying, with the consequence that all death, human and non-human, is merely a banal example of this wider "truth".

This is not a point that Stengers makes. She is interested in the idea that treating the universe as a singular system is only possible because such a universe is not envisaged as having to interact with another environment; and this is why entropy appears to make sense for it "as a whole" ([2], pp. 205-7). But there is a more damning point involved. Considered as a singular whole, technically there is no problem of the "degradation of energy" for such a singular universe. Within this unique and individual universe, "energy is always and everywhere conserved, the extent to which energy is or is not degraded, the extent to which it is or is not usable, depends on the circumstances" ([2], p. 206). To state that, as a whole, the universe is moving toward entropy, toward some kind of equilibrium, is mistaken, for, considered as a whole, the universe is always, in a sense, at equilibrium. But this is only an abstraction. It is only when perspectives are added (and not only human ones) that the question of a move to equilibrium, an increase in entropy, becomes feasible. "The second law of thermodynamics is entirely relative to a cosmological contingency, to the fact that our region of the universe constitutes an improbable local fluctuation" ([2], p. 253). The importance of both human and non-humans perspectives is clear from that fact that "the radiation emitted by the Sun is irreversibly 'dissipated' from the point of view of the Sun but nonetheless 'used' on Earth by every living thing capable of 'exploiting' it" ([2], p. 206).

The above mention of equilibrium in relation to entropy is important. For physics and physical chemistry, entropy becomes a matter of interest when it involves a question of establishing the status of specific gases, liquids, and more, within local, closed-systems (most obviously in laboratories). Such attempts to understand entropy do not seem to be immediately engaged in some teleological conception of the ultimate heat-death of the universe to which all things are drawn. Nevertheless, insofar as the very notion of equilibrium is associated with entropy, it suffers from the danger of its definition being derived from an ideal case, as with Carnot (as outlined above). It turns out that the second law of thermodynamics only makes sense once we have a concept of equilibrium, of a final state, of total equilibrium — but the reason for any actual increase in entropy cannot be provided by thermodynamics itself. "Equilibrium is no longer the thermodynamic "state", but a particular situation within a landscape that asks to be explored" ([3], p. 239).

As becomes clearer in Volume II of Cosmopolitics, it is the work of Ilya Prigogine, with whom Stengers collaborated, that, in her eyes, paves the way for exploring such a landscape. And it is at this point that some of the previous analyses come, hopefully, into sharper focus.

Prigogine is no denier of physics, entropy or the laws of thermodynamics. But he does recognize some of the inconsistencies and problems that have plagued these concepts and which have been all too easily forgotten by physicists and those who accept entropy as describing some inevitable wasting away, some ultimate death. According to Prigogine, the question of entropy is one which is of interest and importance at a "local" level, at a perspectival level, where things actually happen rather than just fade away. There is not time in this article to outline all his thoughts and works (see [3], pp. 105-204) for a fuller account). One important aspect of his stance, however, is as follows:

Prigogine required that physical-chemical processes become a relevant "terrain" for the question of life. He insisted that "irreversibility" - the production of entropy—be able to tie 
its fate not with the evolution toward equilibrium but with the processes that, in one way or another, constitute a living organism ([3], p. 239).

And:

Prigogine had shown that irreversible processes, which produce entropy, can, at the macroscopic scale, be the origin of new regimes of activity that are impossible at or near equilibrium ([3], p. 125)

Rather than view entropy as a matter of mere equilibrium, dissipation, of wasting away, entropy should be viewed as something that is produced. Its production is tied up not with death but with life. This life is not the mere spending of energy, it is "the origin of new regimes of activity". This is not to deny entropy, to wish it away, but it is to give it its rightful place within a wider schema, one in which things happen, far from equilibrium. The sun shines, the plant grows. Life is not a battle against entropy. Life may produce entropy but this is neither its purpose nor its meaning.

The above is only a very brief summary, an indication of a different way of thinking of entropy. It is not one that has been taken up by all physicists and it has not pervaded our culture of thought. To my mind, we remain within a concept of entropy as inextricably linked to wasting away, to the inevitable death of all items of the universe, indeed to the universe itself. This is a point neatly summarized by Stengers:

When we think of the "degradation" of energy, we think of "entropy" [...]. But if entropy is so mysterious, so hard to explain, it is precisely because, as a concept defined [...] only in 1865 , it was not intended to define "energy degradation", or characterise natural processes as a whole. And although it is not incapable of describing the "loss" of available energy and "irreversibility", it does so in a peculiar fashion, without ever abandoning its reference to the ideal defined by Carnot ([2], p. 205).

In the final section of this piece, I will try to outline how this way of thinking affects our thoughts through a discussion of elements of my father's death. Before approaching this, I would like to offer a different conception of the "inevitability" of death, a more hopeful account, as provided in the metaphysical work of Alfred North Whitehead.

\section{Whitehead-Metaphysics and "Perpetual Perishing"}

Having worked as a mathematical physicist, including a book on Einstein, whom he also met, Whitehead was aware of and involved in debates over changing scientific understandings of the "real reality" which 20th century physics claimed to be comprised of atoms, electrons, and so on. However, his aim was not to provide a complete description of the world or the laws of the universe, one which would prove or disprove the concepts of modern physics. Whitehead's major book, Process and Reality [5], is designed to provide a way of thinking that will allow for, even clarify, some of the concepts of physics but is not beholden to them or their presuppositions. He does not want to disallow physics, but he does want to counter its claim that it alone can account for a reality which, almost paradoxically, does not reside in the observable world of pendulums, engines, rocks or sunsets, but in the unobservable "fundamental" subatomic particles which lie "behind" these. 
Whitehead believes in electrons and atoms but insists that there is more to the world than that. Philosophy needs to be able to allow for both electrons and this "more than that". This involves setting out a novel metaphysics and this is the main task of Process and Reality ([5]). To accomplish this, Whitehead invokes a whole host of technical terms such as: "actual entities", "eternal objects", "prehensions". The details and definitions of these are not necessary for the argument being set out here. However, it should be noted that when Whitehead talks of "actual entities", these do not correlate, immediately, to the things of the world as usually conceived - tables, rocks, atoms. Actual entities express the most basic conditions of any moment of existence. This mention of "moment" is instructive. For, as the title of his book suggests, Whitehead is interested in the process of reality. This does not mean that he simply wants to set the universe in motion, like some kind of bad post-modernist; so that all is flow and flux. One main point of the notion of actual entities is to explain the rigid individuality, the atomicity, which is to be found amidst process. They help make up what he calls "stubborn fact" ([5], p. xiii and passim). Such stubborn fact needs to be explained as much as the notion of "process". With this in mind, it is time to turn to the main point that I want to draw out, namely, his use of the phrase "perpetual perishing". This is a term that Whitehead borrows from John Locke but in doing so, he suggests that Locke misunderstood the status and importance of the term.

\section{Perpetual Perishing}

The ancient doctrine that "no one crosses the same river twice" is extended. No thinker thinks twice; and, to put the matter more generally, no subject experiences twice. This is what Locke ought to have meant by his doctrine of time as a "perpetual perishing" ([5], p. 29).

In this passage we have gone quite quickly from the pre-Socratic philosopher, Heraclitus, who saw the world as irredeemably constituted by flow, to a much wider concept of flux. Whitehead has subverted Descartes who stated that there is a thinking thing from which thoughts emanate. According to Whitehead, Descartes got things the wrong way around. The thought comes first and is "a constituent operation in the creation of an occasional thinker" ([5], p. 151). He goes even further. The same applies to every "subject" which experiences. Although he does not make it plain at this stage, Whitehead does not see subjects as limited to the human realm. Every item of existence is, in a very specific way, a subject. However, there is no subjectivity which subtends the experiences of such subjects. Each subject arises anew on each occasion. This expresses the process of reality. Equally, given that there is no thinker or subject hidden behind these experiences, this means that once the experience or thought is complete it is over, it has "died". It is in this way that the process of the world is always accompanied by a "perpetual perishing".

A more "technical" way of putting this would be to talk not of "subjects" but of actual entities. Taken in this light, Whitehead states that: "Actual entities perish, but do not change; they are what they are" ([5], p. 35). This is because actual entities exhibit both the process and stubbornness of reality. "There is a becoming of continuity, but not continuity of becoming [...] extensiveness becomes, but 'becoming' is not itself extensive" ([5], p. 35). Things become what they are. There is process. But, at the metaphysical level, this becoming is not divided. It is one act, one experience, one event. Whitehead cites William James to explain this point, as we experience it at the human level: "Your acquaintance with reality grows literally by buds or drops of perception. Intellectually and on reflection you can divide these components, but as immediately given, they come totally or not at all" ([5], p. 68). Once an experience is 
over, it is possible to divide it up into stages. But while the experience is happening, it comes as one thing. We become surprised or happy, or tired even. Looking back we can, perhaps, identify the steps which went up to make this experience - the room we entered, the person we saw, the work we did. But our experience of, as it occurs, is a unit.

Whitehead extends this idea and states that it applies to all "units of existence" (to all actual entities), and not just to human experience. The corollary of this insistence upon multiple moments of becoming, and the lack of any reliance upon an enduring subject of such experiences, is that each moment is accompanied by a completion, an end, a death. As Whitehead puts it:

time is "perpetually perishing". In the organic philosophy [which is how Whitehead refers to his outlook] an actual entity has "perished" when it is complete. The pragmatic use of the actual entity [...] lies in the future. The creature perishes and is immortal. The actual entities beyond it can say, "It is mine" ([5], pp. 81-82).

Once an experience is complete, it is over and it perishes. "Completion is the perishing of immediacy" ([5], p. 85). This "death" is not without hope. Once an experience is over, once it has become, once an actual entity is complete, it takes its place in the wider world which is also made up of other completed actual entities. This group of actual entities (or "extensive continuum", as Whitehead calls it ([5], pp. 61-82) then becomes the resource, we might even say the "ground", for future occasions of experience, for future actual entities. In this sense, once an experience is over, once an actual entity has become, it is "immortal" ([5], pp. 31-32). Something has happened and nothing can be done about its having happened. What "can be done", however, is that it can be taken up, or not, by future actual entities in their becoming. It is in this way that Whitehead links the past, the present and the future:

This transition, again in Locke's language, is the "perpetually perishing" which is one aspect of the notion of time; and in another aspect the transition is the origination of the present in conformity with the "power" of the past ([5], p. 210).

All this entails that, with regard to "our" world, the one in which humans find themselves, we are accompanied throughout our lives by perishing. "In the temporal world, it is the empirical fact that process entails loss" ([5], p. 340). Yet, perishing, loss, death, do not just apply to humans, or even those things in the world which we think of as living. Every item of existence, each actual entity, may be accompanied by loss but, crucially, this is balanced by the "creativity" which also suffuses existence and is characterized by the very fact that each moment of existence is a becoming of something new.

It is important to take care with this mention of "creativity". This is a term that Whitehead coined. It was not in common usage until he deployed it in the 1920s as a technical philosophical term. Nowadays, we may all too easily take the word to refer to that which is "good" about a person, event, piece of writing, etc. But this is not the role that Whitehead assigns to this term. For him, "creativity" refers to the dynamic, processual aspect of existence. No more no less. It is an inherently neutral term. "Creativity" enables Whitehead to account for novelty (see [6], pp. 35-36 and [7], pp. 134-35), for a fuller discussion of this point). But it is important to remember that there is no moral judgement involved. Cancer exhibits creativity.

Nevertheless, Whitehead accepts that this counterposing of creativity and perpetual perishing presents us with a difficulty, in terms of our human lives: 
The world is thus faced by the paradox [...] it craves for novelty and yet is haunted by terror at the loss of the past, with its familiarities and its loved ones. It seeks escape from time in its character of "perpetually perishing". Part of the joy of the new years is the hope of the old round of seasons, with their stable facts - of friendship, and love, and old association. Yet conjointly with this terror, the present as mere unrelieved preservation of the past assumes the character of a horror of the past, rejection of it, revolt ([5], p. 340).

This is one of the more elegiac passages to be found in Whitehead's text. Unfortunately, it is not the clearest. We dwell amidst a paradox. We crave novelty yet we are haunted by terror at losing the past to which we are accustomed, and this includes those things, situations and people with which we are familiar and that we love. This paradox is reinforced by the realization that novelty and loss are not opposites; they are two sides of the same coin. Novelty requires loss, perishing, death (in the widest sense of this term). This is why New Year is a paradoxical time. We hope for things to continue the same. And yet we look forward to new things. We cannot have both. But Whitehead's point is more subtle than this. For, while we want to retain the certainties of the past, we also have a terror of things remaining the same. Even that which we love is tinged with the horror of the past simply repeating itself. The present would then become a bland repetition of what we have already thought, said, and done.

It is important not to conclude that Whitehead is only making recommendations for understanding human lives and deaths. There are many deaths which inhabit our lives; this is a consequence of the perpetual perishing which accompanies all moments of existence and which enables there to be novelty. These deaths range from the cells of our bodies, to our associations and friendships, to the fleeting moments and situations which make up our lives. These perish so that others can be generated. While such an understanding of our lives and deaths may comprise one element of Whitehead's philosophy, it is important not to lose sight of the more metaphysical aspect. Perishing, loss and death are not limited to humans or living things. They are a condition of all existence. This is balanced by the creativity which is also required for any moment or item of existence. Once again, it should also be noted that Whitehead is no moralist. He provides no framework for establishing what should be retained or saved and what can be lost without any "harm" being done. Nor does he point to which occasions or experiences of the present or future could be worthwhile. To reiterate: cancer is as much an example of creativity as are the paintings of Van Gogh. With this mention of cancer, I will turn to my final section which will try to bring some focus to the previous discussions.

\section{Conclusions}

My father died in September 2013. He had been diagnosed with prostate cancer and Alzheimer's disease about five years previously. Having been admitted to hospital in August 2013 for treatment for an infection, he was discharged toward the end of the month with a package of what was called "end of life care". He had come home to die.

We prepared the downstairs back room of my parents' house for his arrival (this included getting the gas fire disconnected and a safety certificate issued as the fire was "out of date" and could release carbon monoxide which is a health risk to a dying person). My father died on a Saturday. The Sunday before this, the family agreed that he should be put on the Liverpool Care Pathway which is designed, among other things, to enable the provision of pain-killers and sedatives at increased levels without the need for 
a doctor to review or authorise this. A lot of medication is, therefore, made available in the house. These drugs are administered by what is known as a "driver" which is on an automatic timer.

Throughout this article I have outlined two different ways of thinking about death and its supposed inevitability. This involved both a discussion of a (mis-)conception of entropy as derived from physics, followed by a discussion of Whitehead's metaphysical attempt to balance creativity with perpetual perishing. It is not my aim or intention to somehow bring these together into some kind of "theory of death". My point is, rather, to signal that we have inconsistent concepts when it comes to matters of death and dying. Furthermore, we are not always clear what constitutes death. In these final paragraphs, I will not attempt to somehow "solve" these problems but I will try to indicate how some of our difficulties with thinking about death are both informed and limited by ideas which come from other, somewhat surprising, sources. It not so much a matter of providing answers but of changing the kind of questions that we ask of death.

As I mentioned previously, my father had been diagnosed with both prostate cancer and Alzheimer's disease. While a diagnosis of prostate cancer is not to be welcomed, it was accompanied by some kind of hope. We were all involved in a battle with an enemy. It was a battle that might be won, though it might also be lost. If we won, my father would survive, if we lost, he would die. Certain steps, certain treatments, were laid out. Death was a possibility but not, as yet, an inevitability. This was not the case with the diagnosis of Alzheimer's disease. Such a diagnosis seems to come without any hope. According to the medical model, the brain was already dying. Full death was immediately inevitable upon diagnosis. Drugs such as Aricept might slow down the process but it could not halt or reverse it. Unlike the diagnosis of cancer, there was an immediate feeling that whatever the outcome, the battle had already been lost. This approach buys into the unfortunate but widespread view of Alzheimer's disease as representing some kind of living death; a ghostly approach which Moser [8] has summarized as follows:

the idea that people with Alzheimer's disappear into the "mist of oblivion" or some unknown land, that they leave real life reality, and that they get lost to us before they have actually left us. That they are "living dead" ([8], p. 104).

In terms of Whitehead's contrast between creativity and perpetual perishing, one side of this dyad seems to have been forgotten when we talk or think about Alzheimer's disease. The person who has been diagnosed with Alzheimer's disease has been placed solely within the realm of perpetual perishing. The counterbalance of creativity has been omitted. Of course, this is not to be too hopeful or romantic. As stressed earlier, there is nothing inherently good about creativity. Yet, it cannot be ignored, simply because a diagnosis of Alzheimer's has been made. Quite what would constitute a conceptualization of creativity within such situations still needs more work (see [8]), for a review of some possibilities). It is not an easy task, but it is an important one.

I previously mentioned the "driver" to which my father was continuously "hooked up" and which provided a strong pain-killer (Oxycodone) and sedatives, intravenously, in the final days of his life. I think it would be possible to carry out an interesting STS influenced account of the role of the driver as some kind of actant. This is not my intention here. Instead, I would like to focus on the notion of "equilibrium".

My family believed that as the cancer "progressed", my father's pain was likely to be increasing. We aimed to combat this by upping the levels of Oxycodone and sedatives. The idea was to maintain a 
balance. Too much sedative and we would have doped him up unnecessarily. Too little pain-killer and we would be keeping him in a suffering which was unknown to us but well-known for him. The last few days of his life were spent in trying to maintain this balance, or at least to tell ourselves that we were maintaining this balance. We were aiming for a complex equilibrium; one which we tried to maintain in face of the inexorable procession to death. We had accepted this inevitability when we agreed for him to be put on the Liverpool Care Pathway.

It seems possible to read this situation as bearing the hallmarks of the kind of thinking associated with entropy, with the second law of thermodynamics, and the inevitable heat-death of the universe. According to Stengers' [1-3] retracing of this story, entropy, ultimately, can tell us little about the macroscopic world, the kind that my family was facing at that time. And yet, this is precisely how we thought of that aspect of my father's experience. Death was inevitable, what we were aiming for was a "good death" a peaceful one, where a constant equilibrium was achieved. The aim was to avoid any convulsions or violent episodes which signalled a loss of equilibrium on the path to this inevitable death. Put simply, what we were trying to establish was an equilibrium, an on-going entropic state, which balanced the possible increase in pain with an increase in painkillers. This was not an easy task as there was both a gradual increase in pain and a loss of motor function (brought on by the sedative) which meant that the supposedly "closed-system", which my father's body had become, was in constant change.

Maintaining equilibrium in the face of constant and undetectable change is not a simple task. Indeed, it is probably impossible as it presumes infinitesimally small steps in the increase of Oxycodone which manage to maintain an equilibrium while at the same time allowing for change. As Stengers [1] comments, it is one of the "ruses" of Carnot's ideal heat-engine that "it must modify itself in an infinitely progressive manner in order that at no moment will it leave thermal equilibrium" ([1], p. 198). As has been seen, Carnot's heat-engine presumes that the operations involved are reversible. This would seem to imply that death itself is reversible, when it is not. Yet, current conceptions of entropy and equilibrium still rely, tacitly, upon Carnot's thought experiment in order to elaborate the very concept of entropy as an inevitable progression toward heat-death. In terms of my father's final days, we assigned the responsibility for supposedly maintaining a changing equilibrium to a mechanical device, to the driver. And we were happy to do so. It took on the impossible role of the "operator" which "imposes" "the ideally infinitesimal break in equilibrium" ([2], p. 146).

This is one of the reasons that I think that it is important for us to investigate the thoughts (and feelings) which we hold toward death and dying and to trace the extent to which these rely or draw upon concepts which appear to be situated in unrelated fields (physics, for example). As I have suggested toward the end of this piece, Whitehead might offer us a different way of approaching such questions. Clearly he does not offer us any simple answers or solutions. Yet, on the reading of Stengers and Whitehead offered here, it seems clear that the claim of physics that the universe is heading inexorably toward heatdeath only tells of half the story; it talks only of "perpetual perishing". The creativity which is required by any event or moment within this supposedly inexorable path has been missed out. This is not to be too optimistic, however. The word "neoplasm" is often used within medicine to refer to any new form or new growth - as this is the literal meaning of the word. Cancer is then defined as a malignant neoplasm. Nevertheless, all neoplasms are powerfully tied up with novelty; this is what makes them so effective and dangerous to us humans. We need to develop ways of thinking life and death together; 
taking responsibility for both; accepting both; while radically changing what we understand and want out of both life and death.

\section{Conflicts of Interest}

The author declares no conflict of interest.

\section{References}

1. Stengers, Isabelle. Power and Invention. Minneapolis: University of Minnesota Press, 1997.

2. Stengers, Isabelle. Cosmopolitics. Volume I. Minneapolis: University of Minnesota Pres, 2010.

3. Stengers, Isabelle. Cosmopolitics. Volume II. Minneapolis: University of Minnesota Press, 2011.

4. Haraway, Donna, and Ulrike Teubner. Simians, Cyborgs, and Women. The Reinvention of Nature. London: Routledge, 1991.

5. Whitehead, Alfred North, David Ray Griffin, and Donald W. Sherburne. Process and Reality: An Essay in Cosmology (Gifford Lectures Delivered in the University of Edinburgh during the Session 1927-28). Edited by David Ray Griffin and Donald W. Sherburne. New York: The Free Press, 1978.

6. Halewood, Michael. A. N. Whitehead and Social Theory: Tracing a Culture of Thought. London and New York: Anthem Press, 2011.

7. Halewood, Michael. Rethinking the Social through Durkheim, Marx, Weber and Whitehead. London and New York: Anthem Press, 2014.

8. Moser, Ingunn. "Making Alzheimer's disease matter. Enacting, interfering and doing politics of nature." Geoforum 39 (2008): 98-110.

(C) 2015 by the author; licensee MDPI, Basel, Switzerland. This article is an open access article distributed under the terms and conditions of the Creative Commons Attribution license (http://creativecommons.org/licenses/by/4.0/). 